EMERITA. Revista de Lingüística y Filología Clásica (EM)

LXXIV 2, julio-diciembre de 2006

pp. 289-320

ISSN 0013-6662

\title{
PLOTIN ET EPICURE
}

JEAN-MichEL CHARRUE

Les dieux d'Epicure sont beaux, les particules de leurs corps sont transparentes, et les images qu'ils renvoient. Plotin ne semble pas gêné par cette vision où les dieux ne s'occupent pas des affaires des hommes, mais critique l'absence de Providence. La sagesse d'Epicure privilégie le plaisir, ce qui accepté par Plotin avant qu'il ne déclare que le bonheur véritable est le Bien. La matière faite d'atome et de vide, les composés ne lui conviennent guère. La sensation marque un rapprochement de Plotin, avec la théorie épicurienne des images.

Mots clés: Epicure, Plotin, les dieux, la Sagesse, le Bien, le plaisir, le bonheur, la matière, la sensation, les images, la connaissance.
Epicurus' gods are beautiful, their bodies' particles are diaphanous, and the images they send forth. Plotinus does not seem embarassed by this view of gods which does not interfere with men's affairs, but condemns the absence of Providence. The Epicurus' sageness consists to prefer the pleasure, what is accepted by Plotinus, until he said that true happiness is good. Matter formed of atoms, and emptiness, the composed, are not convenient to Plotinus. Sensation will prove some nearness with the theory of eidola.

Key words: Epicurus, Plotinus, the Gods, sageness, the Good, pleasure, happiness, matter, sensation, images, knowledge.

\section{Introduction}

Les dieux d'Epicure sont beaux comme des hommes; les particules de leurs corps transparentes, et les images qu'ils renvoient. De cette vision où les dieux ne s'occupent pas des hommes, Plotin critique l'absence de providence. La sagesse d'Epicure privilégiant le plaisir parait acceptée, avant qu'il ne déclare que le bonheur véritable est le bien. La matière faite d'atomes et de vide, les composés, tout cela ne lui convient guère pas plus que le matérialisme. La sensation marquera un certain rapprochement de Plotin, avec la théorie épicurienne des images, malgré les profondes divergences, même s'il n'en fait pas un instrument de connaissance.

\section{Les dieux}

(a) On peut s'imaginer ces dieux diaphanes, presque transparents, regardant d'une façon si pure et si limpide, impavides, calmes, d'une étrange sérénité, et 
impassibles, dont le corps était fait d'une substance ténue, tenant de la matière, mais d'une texture telle qu'elle leur permettait de vivre éternellement, et dont l'intense bonheur transparaissait dans chacun de leurs gestes, au point qu'ils pouvaient paraître immobiles et en repos, mais qui se mouvaient ${ }^{1}$, d'un mouvement imperceptible pour un regard humain, léger, et d'un autre ordre: «Tels étaient les dieux immortels et bienheureux» ${ }^{2}$.

Et, dans cette ambiance éthérée, d'une lumière diaphane, là où ils étaient les dieux se tenaient droits dans des positions différentes, avec des gestes si mesurés, qu'ils paraissaient dans cette luminosité si intense et si particulière, être d'un autre monde, mais ils avaient des formes humaines, ou presque humaines, c'est à dire qu'ils ressemblaient sans l'être tout à fait, à des hommes d'une étrange beauté, comparables à celles que l'on voit dans l'art et dans la statuaire, si ce n'est qu'ils étaient vivants, car qu'y a-t-il de plus beau et de plus vivant qu'un corps humain $?^{3}$ et de leurs légers mouvements, on s'attendait à autre chose, tant tout semblait si harmonieux, dans leurs traits, les dispositions de leurs membres, leurs proportions isomorphes ${ }^{4}$, et ce qui les différenciait seulement de la beauté humaine, portée à sa perfection, c'était ce regard intensément contemplatif ${ }^{5}$, qui renforçait encore cette divine beauté qu'ils avaient, chacun d'eux, car on pouvait malgré tout les distinguer, quantitativement un par un, dans leur individualité ${ }^{6}$.

Le bonheur des dieux transparaissait ainsi, dans cette sérénité, que l'on se figure, en effet, à partir de celle de l'homme, mais qui là, pouvait atteindre sa réalisation la meilleure: la béatitude des dieux serait ce repos même. Mais en même temps que cette conscience profonde d'être heureux, qui n'existait pas seulement comme simple reprise de l'idée du bonheur, il y avait cette inactivité, cette absence de peine et de fatigue qui d'ordinaire en résulte, par le fait que le bonheur n'est pas seulement dû au hasard de la fortune, mais qu'il était devenu, à la suite de cette prise de conscience, un authentique bonheur intérieur ${ }^{7}$, produit d'une sorte d'énergie morale. Cependant ils n'étaient pas «empêchés pour l'éternité de l'action et d'un faire achevé, du fait même qu'ils avaient cette force

\footnotetext{
Cf. ci-après note 16 , à propos du mouvement des dieux. Epicure, Lettre à Ménécée, 123. Cf. Cicéron, La Nature des dieux, traduction française, Belles Lettres, Paris, 2002, §

4 Cicéron, cit., XVIII, 46 et 50.

Cicéron, cit., XX, 53.

Epicure, Scholie de la Maxime Capitale, 1; cf. ci-après.

Cf. R. Amerio, «L'epicureismo e gli dei», Filosofia 4, 1953, p. 126.
} XVIII, 47.

EMERITA (EM) LXXIV 2, julio-diciembre 2006 pp. 289-320 ISSN 0013-6662 
de discernement» ${ }^{8}$ qui faisait de celle-ci le résultat d'une liberté qui ne trouvait plus d'entraves.

Ils étaient aussi immortels. Ce qui n'est pas sans relation avec la réalisation de leur bonheur, que de le voir se réaliser dans la durée, où leur mort n'aurait pas été sans conséquence sur la vie: aussi ont-ils «un temps infini pour la réalisation de leur plaisir» ${ }^{9}$, et ce, parce que la recherche de l'immortalité les priverait de bonheur ${ }^{10}$. Et on ne saurait tirer argument du fait qu'ils sont composés d'atomes, et que cela les voue fatalement à dépérir ${ }^{11}$, puisque ceux-ci sont remplacés aussitôt par d'autres semblables ${ }^{12}$, ce qui contribue ainsi à l'harmonie de l'ensemble.

On comprend qu'ainsi faits, à cette perfection qui est la leur d'un point de vue physique, se soit jointe la perfection morale, et que les dieux ne dispensent pas pour les hommes le bien et le mal, ainsi que ces derniers le croient, et puissent être de la sorte, de quelque façon que ce soit «la cause de leur malheur puisque de fausses présomptions continuent à leur attribuer, ainsi que la foule les plus grands dommages et les plus grands avantages» ${ }^{13}$. Le fait est que dans l'autosuffisance qui est la leur, ils ne soucient pas des hommes, au contraire de ce que croit la religion populaire.

Les vivants immortels et bienheureux «selon la notion juste que l'on doit avoir d'eux» ${ }^{14}$ sont, en effet, toujours dans cet état d'un plaisir d'une intensité égale à elle-même, que celui soit le plaisir en mouvement ou le plaisir en repos ${ }^{15}$. C'est ainsi qu'on les voit se livrer aux charmes de la conversation dans leur langage et dans cette langue la plus belle qu'est la langue grecque ${ }^{16}$. Et puisque les dieux ont soif, boivent ils, mais ce n'est qu'en respectant cette mesure, et cette perfection qui les empêche de faire un véritable Banquet ${ }^{17}$, et que l'on peut

8 Philodème Sur les dieux, fgt. 44, H.Diels, Philodemos, Über der Götter, III, Philol. Klasse, 1916, nr. 4, p. 57

9 H. Usener, Epicurea, 1887, p. 75, cf. Epicure, Maxime Capitale, 19.

10 Cf..Philodème, Sur les dieux, III, H. Diels, op. cit., fgt. 13, p. 46

11 Cf. Cicéron, cit., XXV, 71.

12 Philodème, Sur la piété, 80, cf. D. Obbink, On piety, 1996.

13 Epicure, Lettre à Ménécée, 124

14 Epicure, Lettre à Ménécée, 123

15 Philodème, Sur les dieux, III, H.Diels,.op. cit., fgt 18, nr. 4, p.49, et nr. 6, commentaire p. 64.

16 Philodème, Sur les dieux, III, H. Diels, op.cit., fgt. 14, nr. 4, p. 37, commentaire, nr. 6. p.50.

17 Philodème, Sur les dieux, III, H. Diels, op. cit., fgt. 76, nr. 4, p.67, commentaire nr 6, p. 80. Diogène Laërce, X, 118 avait montré qu'Epicure avait écrit lui aussi un Banquet, contre le Banquet de Platon.

EMERITA (EM) LXXIV 2, julio-diciembre $2006 \quad$ pp. 289-320 ISSN 0013-6662 
considérer «par hypothèse, qu'ils mangent à la manière des hommes» ${ }^{18}$, mais ce sera une nourriture frugale.

Mais fidèles à l'éloignement qui les caractérise, dès lors qu'ils ne se mêlent pas des affaire humaines, ne sont-ils les instigateurs d'une quelconque providence. Leur état d'ataraxie serait contredit par l'intervention de celle-ci: la fatigue et la peine qui en résulteraient rendent impossible cette action des dieux sur le monde ${ }^{19}$. Lucrèce parle, dans la même perspective que la Lettre à Hérodote, d'une causalité scientifique des phénomènes, à l'exclusion de toute autre intervention, puisque les dieux ne sont pas en mesure de diriger l'univers: «qui pourrait... gouverner l'infini? Faire tourner de concert tous les cieux? $\gg^{20}$. Ce à quoi s'ajoute le fait que la nature n'avait aucune intention ${ }^{21}$, dès lors que la puissance divine est aussi limitée, ne peut-elle, ni ne veut-elle commander le monde ${ }^{22}$. Retrait de la providence qui ne sera pas l'un des moindres éléments de la libération épicurienne.

Chez Plotin, le Principe divin s'appelle l'Un ou le Bien, et présente tous les traits d'une transcendance. C'est aussi à partir de l'idée d'infini qui s'y trouve principalement ${ }^{23}$, se situe toute une série de négations pour l'Un, qui sont fondées sur la première hypothèse du Parménide, en décrivent l'hypostase: se trouvent écartés successivement, le tout et les parties, la figure et la forme, le lieu et l'inhérence, le mouvement et le repos, l'identité, l'égal et l'inégal, la mesure, le nombre, le lieu et le temps ${ }^{24}$, car dans l'infinité même de l'Un, rien de ces caractères qui avaient été dégagés par Platon ne pouvait parvenir à définir sa transcendance.

Certes, parfois Plotin consent à parler de Dieu, comme Zeus, lequel personnifie à la fois le démiurge et l'âme de l'univers, dont il dit: «il doit être le plus doué de mémoire, dans la mesure où il est un démiurge d'une suprême habileté» ${ }^{25}$. Revenir à Zeus, cette fois là, ou dans d'autres cas, c'était revenir à un dieu

\footnotetext{
18 Philodème, Sur les dieux, III, H.Diels, op. cit., fgt. 77, nr 4, p. 67, commentaire, nr 6, p. 84 .

19 Cf.. R. Amerio, «l'epicureismo e gli dei», Filosofia, 4, 1953, p. 110.

20 Lucrèce, De la Nature, II, 1096-1097, cité par A. Gigandet, «Providence et cause finale, une polémique épicurienne», dans L'épicurisme antique, Cahiers philosophiques de Strasbourg, 2003, p. 57.

21 Lucrèce, op. cit., II, 181, cf. G. Rodis-Lewis, Epicure et son école, Paris, 1975, p. 73.

22 Lactance, Colère de Dieu, 13, 19.

23 Ennéades, V, 5, 1, 4; VI, 7 (38), 32, 15; VI, 9 (9), 6, 10; VI, 5 (23), 12, 5-11.

24 Cf. J.-M. Charrue, Plotin lecteur de Platon, Paris, 1978, les tableaux, p. 64-74.

25 Ennéades, IV, 4 (28), 9, 9, cf. J. Pépin, «Dieu est-il tout puissant, Alexandre, Origène
}

EMERITA (EM) LXXIV 2, julio-diciembre $2006 \quad$ pp. 289-320 ISSN 0013-6662 
de la tradition hellénique, mais était-ce la majorité des textes, et l'ensemble d'une description de Dieu, qui pour l'essentiel se situait au niveau de l'Un ou du Bien, c'est à dire d'un Dieu impersonnel?

D'autres figures que celles de Zeus apparaissent dans les Ennéades, ainsi celle d'Héraclès qui représente l'âme divine libérée du sensible ${ }^{26}$, le mythe d'Erôs, occupe Tout l'espace de certains traités comme III, 5, ou l'histoire du miroir brisé du jeune Dionysos, montrant cette dispersion des consciences ${ }^{27}$. On peut considérer que Plotin a reçu le mythe, dans la plus pure tradition de l'hellénisme, qu'il en a tiré toutes les leçons. Chez lui, les dieux se mêlent aussi des histoire des hommes: Ulysse, malgré toutes les plaisirs des yeux, et toutes les beautés sensibles s'est échappé pour retourner vers sa vraie patrie ${ }^{28}$. Contrairement à Epicure, Plotin appréciait Homère et en réécrirait parfois l'histoire ${ }^{29}$.

Pourtant, il est un passage du traité III, 2, De la Providence, qui le voit près des dieux d'Epicure, lorsqu' il déclare: «les dieux n'ont pas à négliger leur propre vie pour régler nos affaires particulières» ${ }^{30}$. On retrouve là cette transcendance des dieux épicuriens qui ne s'occupent pas des affaires des hommes, parce qu'ils ne s'occupent pas du bien et du mal, dont ils ne sont pas pour eux, la cause. Sans doute la hauteur des dieux épicuriens, convenait-elle, dans une certaine mesure à l'idée de transcendance dont il était parti.

Aussi se les figurera-t-il un peu à l'image d'Epicure qui n'est peut-être pas absent de cette description: «Les êtres bienheureux sont immobiles en eux-mêmes - c'est l'idée d'un bonheur résidant dans le repos qu'on s'en faisait parfois -; ils leur suffit d'être ce qu'ils sont; ils ne se risquent pas à s'occuper de tout; ce serait sortir de leur état. Mais telle est leur félicité que sans agir, ils font pourtant de grandes choses, et qu'ils produisent beaucoup en restant immobiles» ${ }^{31}$. Quel plus bel hommage aux dieux d'Epicure que l'on a peint contemplatifs, dont la contemplation rejoint la contemplation plotinienne, qui on le sait produit, ainsi

et Plotin», The Perennial tradition of neoplatonism, Louvain, 1997, p. 14

26 Ennéades, IV, 3 (27), 27, 1-14.

27 Cf. notre article «Plotin et le désir», à paraître dans Symbolae Osloenses, note 58 et Ennéades, I, 4 (46), 10, 11.

28 Ennéades, I, 6 (1), 8, 16-20.

29 Nous projetions de commencer, à propos des dieux par ce débit de l'Odyssée d'Homère, I, 32-33: (Zeus): «Ah misère! Ecoutez les mortels mettre en cause les dieux! C'est de nous, disent-ils que leur viennent les maux...». Nous nous demandons si Epicure n'a pas été une sorte de réponse à cette inquiétude de Zeus, et s'il n'a pas tout simplement fait en sorte qu'il ne puisse plus y avoir ce mal des dieux pour les hommes.

30 Ennéades III, 2 (47), 9, 12-13.

31 Ennéades III, 2b (47), 1, 40-45.

EMERITA (EM) LXXIV 2, julio-diciembre $2006 \quad$ pp. 289-320 ISSN 0013-6662 
qu'il apparaît dans le traité III, 8, De la contemplation: où Plotin rejoint ainsi, dans ce moment là, les dieux d'Epicure.

Jusqu'au moment où il s'agit de la Providence dont il reproche aux épicuriens l'abandon: «l'ordre ne naît pas du désordre, ni la loi de l'inégalité, comme le croit certain philosophe» ${ }^{32}$. Le principe de l'ordre, c'est la providence qui s'étend à tout et gouverne le monde: «le principe producteur de l'univers ne doit jamais être en peine, bien que certains aient pensé que le gouvernement du monde est un travail pénible» ${ }^{33}$. La providence est ainsi cet ordre qui s'étend à tout en tant que principe rationnel, dont il consacre la présence de dieu en l'intelligence, de celle-ci en l'âme, et à partir de l'âme consacre la réalité à tous les niveaux ${ }^{34}$. «Intelligence extérieure à notre monde sensible, d'où procède notre univers et selon lequel il existe», bel ordre que les épicuriens ont voulu détruire, en sorte qu'ils ne peuvent qu'être désavoués, et que la critique doit se faire plus vive: «Epicure nie la providence et nous conseille de rechercher à nous complaire dans les plaisirs» ${ }^{35}$. C'est la seule fois où le nom est prononcé, et c'est là; c'est dire l'intérêt de Plotin, pour la question.

B La scolie de la Maxime Capitale s'énonce ainsi: «Ailleurs il dit que les dieux ne peuvent être vus que par l'esprit; les uns existeraient comme individualités discernables, les autres présenteraient entre eux une ressemblance parfaite à cause du flux incessant de simulacres, et tous auraient forme humaine» ${ }^{36}$.

Cette scolie un posé un nombre considérable de problèmes, dont on trouve un résumé dans la Pensée du Plaisir de J. Bollack ${ }^{37}$. Son intérêt étant de faire comprendre toute l'épistémologie épicurienne sur la connaissance des dieux. Le premier point paraît, à l'évidence, une allusion à la "prénotion" épicurienne ou "prolepse" ${ }^{38}$ qui était le point de départ de toute connaissance, en tant qu'idée commune et préalable de la pensée des dieux.

32 Ennéades III, 2 (47) 4, 27-28, l'allusion porte sur la naissance d'une nécessité et d'une ordonnance du monde à partir du hasard, du déplacement des atomes, cf. Lettre à Hérodote, 43 sur les atomes en mouvement perpétuel.

33 Ennéades, IV, 4, 12, 40= Usener, 352.

34 J. Trouillard, «La présence de Dieu selon Plotin», Revue de métaphysique et de morale, 59, 1954, p. 38-45.

35 Ennéades, II,9 (33), 15, 8-9.

36 Epicure, Scolie de la Maxime capitale I, nous l'avons présentée dans la traduction d'Hamelin, Lettres,édition Nathan, en note p. 112, qui pose le moins de problème, même si c'est plus une interprétation qu'une traduction..

37 J. Bollack, La Pensée du plaisir, Paris, 1975, p. 217-238.

38 Epicure, Lettre à Ménécée, 124. 
Mais, partant de là, nous sommes confrontés, à une théorie des images comme perception, à partir de leur flux continuel. Le texte de Philodème, Sur les dieux, à partir d'une interprétation probablement erronée de Diels ${ }^{39}$, éclaire ce texte: il n'y a pas deux types de dieux comme il le croyait, les uns reconnaissables un à un, les autres identifiables aux astres, puisqu'il n'y a pas de divinité astrale pour Epicure. Mais si les premiers sont nombrables $\kappa \alpha \tau$ ' $\alpha$ pi $\theta \mu$ òv, que l'on peut interpréter pris individuellement ${ }^{40}$, les autres ne le sont pas, parce les images émanant de leurs corps pour la perception, se sont mêlées ainsi que l'indique Philodème, à celle des astres ${ }^{41}$.

Remarquable théorie de la perception, où les images qui se détachent sans cesse des dieux, et nous les font connaître indiquent aussi que les dieux vivaient dans des inter mondes et dans un autre lieu, en raison même de leur consistance, et qu'ils ne pouvaient vivre sur la terre ${ }^{42}$, tandis que leurs images pouvaient, à partir de ce flux, être perçues par nous.

Toute autre est la conception plotinienne de la vision des dieux. Dans le traité Sur le Bien ou sur l'Un, l'âme ne peut voir l'Un que par une vision intelligible: «Quand elle veut voir par elle-même, alors elle le voit seulement par le fait d'être unie à lui» ${ }^{43}$. On est aux antipodes d'Epicure, car, dans cette vision, quand on a vu en quelque sorte, comme s'il était une chose sensible, afin d'en avoir une représentation....», dans les mouvements immobiles et le repos, «les choses ne se distinguent pas une à une, et pourtant ne se confondent pas, car chacun se déploie en se distinguant des autres» ${ }^{44}$. On ne saurait dire cependant qu'il y a quelque chose de comparable à la vision $\kappa \alpha \tau^{\prime}$ ảpi $\theta \mu$ òv, même si le texte a des allures de réplique à la théologie d'Epicure. Plotin a pu peut-être plus s'inspirer de la vision des mystères «là où, dans le sanctuaire après l'objet de la vision, qu'il a contemplé, et le commerce non pas avec une statue, une image, mais avec Lui» ${ }^{45}$.

Certes, dans d'autres textes, l'on voit des dieux multiples: «Tel est ce dieu

39 H. Diels, op. cit., nr 6, en commentaire, p. 25.

40 J. Bollack, op. cit., p. 217 traduit« les uns suivant le nombre», ce qui est une traduction; mais c'est incompréhensible, à notre sens, c'est pourquoi nous notons «pris individuellement», ayant ajouté: ce n'est pas une traduction -littérale s'entend-, c'est plus une interprétation; on pourrait traduire «nombrables»

${ }_{41}$ Cf. à ce sujet G. Arrighetti, «Sul problema dei tipi divini nel’ epicureismo», La Parola del Passato, 10, 1955, p. 412-413.

42 Philodème, Sur les dieux, H. Diels, op. cit., nr 4, p. 26.

43 Ennéades, VI, 9 (9), 3,11-12, 1994, tr. P. Hadot.

44 Ennéades, VI, 9 (9), 5, 12-13.

45 Ennéades VI, 9 (9), 9, 10, 20-21. 
multiple, il existe en l'âme qui est attachée à lui» ${ }^{46}$. Sans doute y a-t-il parfois «des dieux intelligibles» ${ }^{47}$, mais «le Dieu ne se divise pas, puisqu'il n'est pas en un lieu et est immobile» ${ }^{48}$. En fait, Plotin veut écarter tous ceux qui pensent que la vision de Dieu puisse être un objet matériel: «Lorsque vous le voyez, regardez le tout entier ... et pensez qu'il est le Bien» ${ }^{49}$.

La sagesse

«Tu vivras comme un dieu parmi les hommes», conclut le Lettre à Ménécée $e^{50}$ : c'est là le devenir promis au sage. Aussi cette sagesse présente t-elle ce trait commun à toutes les sagesses antiques depuis le kalos kagathos de Platon, d'un certain esthétisme extérieur. Le fait est que le texte est précédé dans Diogène Laërce, d'un Portrait du sage ${ }^{51}$ qui s'efforce d'en présenter les traits extérieurs et intérieurs, où l'on apprend que le sage «se doit de rejeter la haine, l'envie et le mépris» ${ }^{52}$ qui en sont les caractéristiques premières, et que faisant fi du siège continuel des passions qui l'assaillent ${ }^{53}$, il s'appuiera sur ses amis et contrairement au stoïcien gémira dans la torture ${ }^{54}$.

Description qui trouve son complément dans la Lettre à Ménécée, lorsqu'il

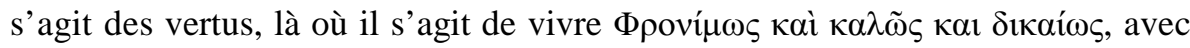
prudence, honnêteté et justice, et ce, de façon à ne jamais éprouver de honte, où l'on voit combien cette morale s'éprouvait de l'extérieur. Sénèque le rendra en disant que «c'est un précepte d'Epicurequ'il faut faire le choix d'un homme de bien, et de l'avoir constamment sous les yeux, de manière à vivre comme sous le regard, et à régler toutes nos actions comme s'il les voyait» ${ }^{55}$, de vivre ainsi

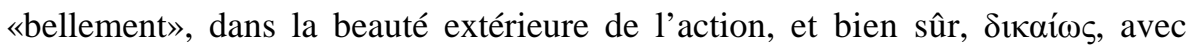
justice.

Les trois qualificatifs constituent ensemble, ce qu'Epicure a pu dire de la ver-

46 Ennéades $\mathrm{V}, 1(10), 5,1$.

47 Ennéades V, 1 (10), 7, 29-30.

48 Ennéades V, 1 (10), 11, 7-8.

49 Ennéades V, 5 (32), 10, 10, cf. E. Bréhier, Notice à V, 5, p.88.

Epicure, Lettre à Ménécée, 135.

Diogène Laërce, X, 117-121, cf. J. Bollack, op. cit., pp. 22-31.

52 Epicure, Le Sage, Diogène Laërce, X, 117, 5-6, J. Bollack, op. cit., pp. 22-23.

53 Epicure, op. cit., 117, 7-10.

54 Epicure, op. cit., 118, 1-5.

55 Sénèque, Lettre 11, 8, repris dans Usener, Epicurea, cf. M. Conche, Epicure, Lettres et Maximes, 1990, p. 76.

EMERITA (EM) LXXIV 2, julio-diciembre 2006 pp. 289-320 ISSN 0013-6662 
tu; il est vrai qu'il n'en a parlé que rarement ${ }^{56}$, mais aux endroits clés de sa pensée; dans la Maxime Capitale 5, il ajoutera: «Il n'est pas possible de vivre avec plaisir sans vivre avec prudence, honnêteté et justice, ni de vivre avec prudence, honnêteté et justice, sans vivre avec plaisir» ${ }^{57}$, rendant la proposition converse tout aussi vraie.

C'est sur ce point que les stoïciens, et Cicéron s'attaquaient au troisième

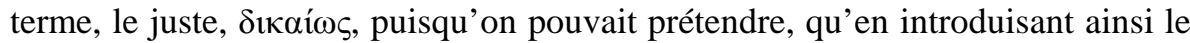
plaisir, à côté de la justice, on pouvait transgresser celle-ci, à son profit, et ainsi arriver à l'injustice, commettant des actions mauvaises sans que cela se sache, avec l'impunité, et que de la sorte la justice ne serait digne de choix que si elle procure du plaisir, ce qui la rend tributaire de celui-ci, contredisant la morale. Mais l'ataraxie morale ne préserve telle pas d'un tel travers? ${ }^{58}$

Les trois vertus étaient liées et contribuaient à la réalisation de la sagesse. $\mathrm{Ce}$ qui était bien sûr le cas de la prudence ou sagesse pratique. Celle-ci se présente dans l'évaluation même des désirs, puisqu'elle était à même de réfléchir sur le bien et le mal, de voir les limites où les excès pouvaient conduire: «Là où Epicure est excellent, c'est quand il dit que les réduisant, le sage traverse les infortunes, et que les choses les plus importantes, sont administrées par lui, en usant de sa propre réflexion et de son calcul» ${ }^{59}$.

La sagesse était vouée à devenir pratique, parce qu'elle était une activité de

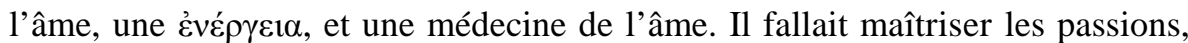
plaisirs et douleurs, afin d'arriver à leur suspension, à cet état d'ataraxie, mais, pour ce faire, quitter cet ordre de la passivité, tendre vers un principe d'action où intervenait la volonté à la suite du jugement. C'était le rôle de la sagesse: elle s'adressait à un homme libre et lui prodiguait des conseils. On est étonné de voir à quel point ceux-ci étaient pratiques: «le sage évitera l'union (le mariage) à une femme, ne punira pas les domestiques, n'éprouvera pas la passion de l'amour, ne se souciera pas de sépulture, ne croira pas que la passion est envoyée par les dieux...» ${ }^{60}$. On apprenait par cœur, dans les écoles épicuriennes, ces choses là:

56 Cf. la critique de Cicéron, Des termes extrêmes des biens et des maux, (= De finibus), II, XVI, 51. 251.

7 Epicure, Maxime Capitale 5, tr. M. Conche, op. cit., p. 233; J. Bollack, op. cit., p.

58 Cf. P. A. Van der Waerdt, «The justice of epicurean wise man», Classical Quarterly, 37, 1987, p. 402, et J. Salem, Tel un dieu parmi les hommes, l'éthique d'Epicure, Paris, 1989, p. 104.

59 Cicéron, cit., I, XIX, 62.

60 Diogène Laërce, X, 118-121, J. Bollack, op. cit., pp. 24-31.

EMERITA (EM) LXXIV 2, julio-diciembre $2006 \quad$ pp. 289-320 ISSN 0013-6662 
«Il fallait s'exercer à méditer quotidiennement la regulae uitae qui consiste en un petit nombre de dogmes. Les avoir présent à l'esprit était plus important que le savoir philosophique le plus détaillé et plus minutieux» ${ }^{61}$. Le second socratisme était né. Les écrits de Philodème montrent bien qu'on avait tiré les leçons de l'échec du premier: la vertu, certes, ne s'enseigne pas, mais on pouvait l'élargir à la vertu en général, laquelle ne pouvait être simulée, se consacrer davantage à la pratique, par exemple aux travaux des champs, à l'amour de la campagne qui ne pouvaient que renforcer la vertu ${ }^{62}$. Et la philosophie pouvait être enseignée au peuple.

Et là aussi allait-il vers plus de sagesse, cette sagesse qui s'organisait autour de la notion de limite ${ }^{63}$; nous dirons de mesure pour rester fidèle à l'esprit de la Lettre à Ménécée. L'Erôs socratique et platonicien avait introduit la démesure, ne serait-ce que comme conséquence. L'amitié était plus simple: née du besoin, elle était utile, autre trait de la morale socratique, mais cette utilité ne la pervertit pas, au contraire: «L'amitié naît de l'usage: il faut toutefois qu'elle ait commencé antérieurement; de fait nous ensemençons la terre. Mais elle prend consistance dans la communauté, dans ceux qui ont été comblé par les plaisirs» ${ }^{64}$. Ainsi peu à peu se fait-elle désintéressée: «les épicuriens se portaient secours dans les malheurs publics» ${ }^{65}$. Ainsi peut-on considérer qu'elle constituait sur le plan social, cette quatrième vertu ${ }^{66}$. «Le sage s'appuie sur ses amis, quand ils présents ou qu'ils sont absents» ${ }^{67}$; «pour défendre son ami il peut lui arriver de perdre la vie» ${ }^{68}$. Tant est fausse l'idée d'un individualisme d'Epicure, semblable à ces atomes isolés du monde ${ }^{69}$. Le désaveu du politique avait les raisons que l'on a dites, mais il était remplacé par cette vertu nouvelle, d'une amitié qui pouvait constituer de la sorte la base du renouveau d'une communauté, dans cette vie en commun simple et prosaïque qu'elle inaugurait.

${ }^{61}$ I. Hadot, «Epicure et l'enseignement philosophique hellénistique et romain», VIIIè Congrès Budé, 5-10 Avril, 1968, pp. 350-352.

${ }_{62}$ E. Acosta-Mendez et A. Angeli, Filodemo, Testimonianze su Socrate, Naples, 1992, fgt 18, commentaire, p. 275, tiré de Philodème, Rhétorique VII, Papyrus Herculanum 1669; cf. fgt. 32, commentaire, p. 361, Papyrus Herculanum. 1424

63 Cf. G. Romeyer-Dherbey, «Epicure le philosophe qui vivait dans un jardin», en préface à la réédition de J. M. Guyau, La morale d'Epicure, Paris, 2002, p. 13.

64 Diogène Laërce, X, 120b 43, Portrait du sage, J. Bollack, op. cit., p. 31.

65 Cicéron, Des termes extrêmes des biens et des maux, (= De finibus, ), II, XXX, 101.

66 Cicéron, op. cit., II, 66.

67 Diogène Laërce, X, 118, cf. J. Bollack, op. cit., p.25.

68 Diogène Laërce, X, 120B, J. Bollack, op. cit., p. 29.

69 Nous nous démarquons ici de V. Goldschmidt, La doctrine d'Epicure et le droit, Vrin, Paris, 1977, p. 47. 
Ainsi la sagesse avait-elle pour fin le bonheur. Dans la Lettre à Ménécée, il affirme d'entrée: «il faut donc méditer sur ce qui nous procure le bonheur, puisque lui présent nous avons tout, et lui absent, nous faisons tout pour l'avoir» ${ }^{70}$. Seulement, il faut savoir dominer le temps, anticiper le bonheur futur, pour le jeune, revoir le bonheur passé, pour le vieux. ${ }^{71}$ Seule l'âme peut le faire. Ainsi devient-il principe de vie, fin de toute la vie et de la sagesse. Seulement, dès qu'il s'agit de le définir, Epicure pense que c'est le plaisir seul, le plaisir du corps, puis celui de l'âme. «Le plaisir est le principe et la fin de la vie heureuse» ${ }^{72}$, c'est à dire, aussi, son commencement: «le bien premier et connaturel» ${ }^{73}$.

«Epicure affirme que le souverain bien est le plaisir», note Cicéron ${ }^{74}$, et de se récrier, comme à peu près tout le monde, comme s'il n' y avait pas la cette protestation contre les idéaux factices et les biens illusoires qu'on proposait le plus souvent à l'humanité. Epicure, comme sage a pris conscience d'une vacuité possible du Bien, d'une extériorité de celui-ci, pour ne partir que du plaisir le plus simple, le plaisir corporel. Seul celui-ci existe. Ce plaisir là est constitutif, catastématique, comme il dit, c'est à dire constitutif d'une santé du corps, sans laquelle il ne serait pas possible d'être heureux ${ }^{75}$. Après la souffrance, le trouble, la maladie, le départ de celle-ci déjà, à lui seul, constitue le plaisir: c'est ce qu'il appelle le plaisir en repos. Un peu comme s'il avait dit le bonheur, c'est cette santé, et rien n'est possible sans elle, le bonheur est la santé du corps: et c' est déjà un plaisir que de retrouver ainsi le repos, aponie et ataraxie, absence de peine pour le corps, de trouble pour l'âme: «c'est pour cela que nous faisons tout pour ne pas souffrir, et ne pas être troublé. Une fois cela réalisé en nous, toute la tempête de l'âme s'apaise» ${ }^{76}$.

A côté de cela: un autre qui va vers les plaisirs, le plaisir en mouvement. Mais il s'agira pour l'un comme pour l'autre de savoir choisir, et c'est là le début de toute sagesse entre les désirs naturels et nécessaires pour le bonheur, pour la vie, pour le bien-être du corps, ceux simplement naturels, enfin les désirs vains ${ }^{77}$. Toute la sagesse consiste dans ce choix fait par l'âme entre les plaisirs.

70 Epicure, Lettre à Ménécée, 122.

71 Epicure, Ibid.

Epicure, Lettre à Ménécée, 128.

Epicure, Lettre à Ménécée, 129.

74 Cicéron, Des termes extrêmes des biens et des maux (= De finibus), I, 9.

75 Sur cette, cf. V. Brochard, «La théorie du plaisir d'après Epicure», Etude de philosophie ancienne et de philosophie moderne, 1974, pp. 269-270.

76 Epicure, Lettre à Ménécée, 128.

77 Epicure, Lettre à Ménécée, 127.

EMERITA (EM) LXXIV 2, julio-diciembre 2006 pp. 289-320 ISSN 0013-6662 
Epicure a beau nuancer: «quand nous disons que le plaisir est la fin, nous ne parlons pas des plaisirs des gens dissolus, et de ceux qui résident dans la jouissance comme le croient certains qui ignorent la doctrine» ${ }^{78}$. Il a soulevé un torrent de protestations, parce qu'il avait dérangé. Les plaisirs ont beau n'être parfois pas ceux du corps, mais ceux de l'âme: plaisir de la pensée à côté du plaisir de la chair ${ }^{79}$, qu'il est impossible de dissocier parce qu'il faut connaître la nature et le monde physique, au préalable, ce qui, en soi, assure à la vie sa parfaite sérénité $^{80}$. Mais ce plaisir spirituel restait, uni, malgré les variations, $\pi$ oıкí $\lambda \mu \alpha \tau \alpha$, dans l'unité du plaisir, capacité de l'être vivant, santé du corps et santé de l'âme.

Epicure est un libérateur: de même qu'il avait libéré les hommes de toutes les craintes qui rendaient leur vie impossible, celle des dieux, de la mort, il avait libéré les hommes des désirs futiles, lui avait redonné une certaine autonomie intérieure, qui était celle du sage; on lui saura gré par la suite, d'avoir rendu possible une vie agréable, une vie que l'on s'est choisi ${ }^{81}$. Liberté intérieure du sage qui a réduit les trouble et trouvé la sérénité, et liberté extérieure vis à vis de la religion ou d'autres exigences. Sans doute y a t-il négation de certaines valeurs, mais derrière le Bien on ne savait pas ce qu' on allait imposer à l'existence humaine: en niant cette transcendance, instrument possible de certains asservissements, il avait tout simplement rendu à l'homme sa liberté.

Plotin a t-il développé sur la sagesse une réflexion proche de celle d'Epicure? A priori les deux pensées restent sur ce point fort différentes, voire opposées. S'il l'a fait une fois, on peut s'attendre à ce que ce soit dans le traité Sur le Bonheur, puisque c'est quand il traite une question qu'il en vient dans son parcours dialectique à explorer les possibilités et à s'interroger sur les thèses en présence.

Or, on le voit très précisément, au début du traité I, 4 (46), côtoyer l'épicurisme, et ce par le fait même d'essayer de mettre une étiquette sur celui-ci, et d'envisager l'hypothèse du plaisir: «si la fin des biens est le plaisir, et si bien vivre consiste à éprouver du plaisir» ${ }^{82}$. Autrement dit, la solution épicurienne est-elle envisageable? ce n'est pas en toutes lettres, mais c'en est comme la trame du questionnement qui inaugure le traité, ainsi que cette insistance sur le mot

78 Epicure, Lettre à Ménécée, 131.

79 Epicure, Lettre à Ménécée, 144.

80 Epicure, Lettre à Hérodote, 37.

81 M. Pohlenz, La liberté grecque, tr. fr., Paris, 1956, pp. 180-186, cite l'exemple d'Horace qui «loue Epicure d'avoir permis l'existence confortable de la vie», et Cicéron qu' « il a permis de vivre comme l'on veut».

82 Ennéades, I, 4 (46), 1, 25-26.

EMERITA (EM) LXXIV 2, julio-diciembre $2006 \quad$ pp. 289-320 ISSN 0013-6662 


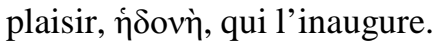

Ainsi la question rebondira-t-elle plus loin, dans cette réitération du questionnement, qui lui est coutumier, de savoir si le bonheur c'est le plaisir, encore faudra-t-il reconnaître que «le plaisir est un bien» ${ }^{83}$, où la solution arrive un peu après: «le bonheur appartient non pas à l'être qui sent le plaisir, mais à celui qui est capable de connaître que le plaisir est un bien» ${ }^{84}$. C'est dire d'une façon différente: le sage (épicurien) est-il à même d'avoir la mesure du bonheur, puisqu'en effet, c'est lui qui fait le diagnostic, le plaisir c'est le bien?

Le mot est volontairement emprunté au jargon médical: Epicure faisait de la philosophie une médecine de l'âme, et il sera question, bien plus avant, «du plaisir, de la santé, et de l'absence de peine» ${ }^{85}$, ce qui indique qu'il ne perdra pas, en cette fin de traité, la position initiale. Il n'est que la problématique du vivant qui ne soit un écho à Epicure, lorsque celui-ci envisageait les plaisirs catastématiques, c'est à dire constitutifs, qui restaurent le repos après le trouble, qui ne soit envisageable. Comme si Plotin s'était demandé, mais le plaisir est-ce la santé du corps? Mais, au début, quand il demande si le plaisir qui peut procurer le bonheur, il est amené à situer celui-ci à l'intérieur du vivant. Le plaisir serait alors la vie, l'éclosion de celle-ci. Alors pourquoi ne pas l'accorder aux animaux: les oiseaux chanteurs ou les plantes ${ }^{86}$, dans cette généralité d'une vie qui s'étend?

Ainsi le bonheur serait le plaisir, parce qu'il s'étend au bien vivre ${ }^{87}$ qui était propre à Epicure ${ }^{88}$, et qui plus est, ce bien vivre consistait dans l'ataraxie évoquée par Plotin ${ }^{89}$, ou dans une conformité à la nature. On ne saurait être plus proche de la problématique épicurienne. Aussi a-t-on pu voir, derrière le sage plotinien, se profiler le sage épicurien ${ }^{90}$, ce qui apparaît des plus justes si l'on suit les termes de cette citation: «celui qui possède ce principe vivifiant mène

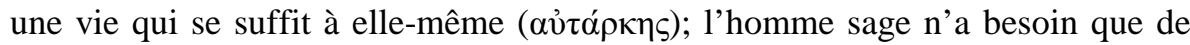
lui-même pour être heureux et acquérir le bien; il n'est de bien qu'il ne possède. Il cherche d'autres choses, c'est vrai; Mais il les cherche parce qu'elles sont indispensables, non pas à lui; mais aux choses qui lui appartiennent; un corps lui

83 Ennéades, I, 4 (46), 2, 19.

84 Ennéades, I, 4(46), 2, 22-23.

85 Ennéades, I, 4 (46), 14, 28.

86 Ennéades I, 4 (46), 1, 20-23; et ligne 8.

87 Ennéades I, 4 (46), 1, 27 et 29.

88 A.Schniewind, L'éthique du sage chez Plotin, Le paradigme du spoudaios, Vrin, Paris, 2003, p. 73.

89 Ennéades, I, 4 (46), 1, 28.

90 M.Tortorelli Ghidini, «L'ambigua presenza di Epicuro in Plotino», in Epicureismo greco e romano, 1996, II, p. 991.

EMERITA (EM) LXXIV 2, julio-diciembre $2006 \quad$ pp. 289-320 ISSN 0013-6662 
est uni, et il les cherche pour ce corps; ce corps lui aussi, est un être vivant, mais vivant d'une vie qui a ses biens propres qui ne sont pas ceux de l'homme véritable. L'homme connaît ces biens du corps, et les lui donne sans rien entamer de sa vie à lui» ${ }^{91}$. Le texte est exemplaire pour situer un point de départ épicurien, équivalent à la reconnaissance des désirs nécessaires pour la vie et pour le bienêtre du corps, dans la Lettre à Ménécée, et ce, même pour le sage ${ }^{92}$, avant toute autre chose, ou comme condition d'autre chose, qui marquera cette fois, l'entrée dans une position différente de Plotin.

On a reconnu au passage l'autarcie qui caractérisait le sage épicurien ${ }^{93}$, et le fait que le désir donnant lieu au plaisir trouve son origine dans le corps vivant, ce que Plotin note ailleurs: «le plaisir au lieu de la peine, la satisfaction au lieu du besoin,ce n'est pas l'âme qui recherche les saveurs douces ou amères, c'est le corps ${ }^{94}$. Le reconnaître, c'était accepter les désirs du corps, et un passage obligé, même pour le sage, qui lui aussi est un être physique, par les désirs corporels, même s'il n'est que momentané, puisque Plotin voudra aller vers d'autres choses.

Mais il tend à accorder, à cet instant, une place au plaisir: «Et ses souffrances personnelles - il les supportera tant qu'il pourra; lorsqu'elles dépassent la mesure, elles l'emporteront. Il n'excitera pas la pitié par ses souffrances; la flamme qui est en lui brille comme la lumière de la lanterne dans les tourbillons violents des vents et de la tempête» ${ }^{95}$. Il essaie de retrouver dans les troubles la sérénité de l'âme. La douceur du plaisir retrouvé par delà les souffrances serait alors le bonheur. Il n'est que l'amitié, dans le traité qui ne participe à celle-ci: «Le sage éprouve pourtant les sentiments d'amitié et de reconnaissance, et il les éprouve à son propre égard; il se rend à lui-même tout ce qu'il se doit; il témoigne de l'amitié à ses amis, mais d'une amitié accompagnée de clairvoyance intellectuelle» ${ }^{96}$.

Il a fui les idées de la mort vers lesquelles le conduisaient ses souffrances et les souffrances d'autrui ${ }^{97}$, retrouvant avec cette sérénité le bonheur, lorsqu'il s'en est libéré, mais la question primitive demeure: le plaisir est un bien, mais

91 Ennéades, I, 4 (46), 4, 24-30.

92 A., Schniewind, Ibid.

Epicure, Lettre à Ménécée, 130.

94 Ennéades IV, 4 (28), 20, 22-28, et 4-5.

95 Ennéades I, 4 (46), 8, 1-5; M. Tortorelli Ghidini, art. cit., pp.993-994, voit à la fin un souvenir du célèbre passage de Lucrèce.

96 Ennéades I, 4 (46), 15, 21-25.

97 Ennéades I, 4 (46), 8, 12. 
est-ce le Bien ${ }^{98}$ ? C'est ce que Plotin ne peut reconnaître. Le Bien moral, les vertus sont-elles des biens liés à chaque chose, à chaque affection; n'y a t-il pas un seul bien, le vrai Bien? «Si le bonheur est placé dans la possession du vrai bien, pourquoi l'oublier?» ${ }^{99} \mathrm{C}$ 'est alors que Plotin s'éloigne délibérément des données de l'épicurisme, rompt avec celui-ci: il y a un autre bien que le plaisir, et ce Bien moral et transcendant l'emporte sur tous les autres. Dès lors, le sage s'efforcera, après avoir évité les maux, d'aller vers ce bonheur plus positif, plus essentiel, celui du Bien, vers lequel il tendra de toute sa volonté.

Dans le traité I, 5 Le bonheur s'accroît il avec le temps, il objectera encore: «En quoi le souvenir du plaisir est-il agréable? Qu'y a t-il d'agréable à se rappeler qu'hier on a pris un bon dîner?» ${ }^{100}$. La perte de ses parents et amis n'émeut en lui que la partie irrationnelle de l'âme dont les peines ne l'affligent guère ${ }^{101}$, un peu comme «ceux qui n'ont pas gémi, comme si leur sort était digne de pitié, sur ceux qui ont perdu la vie» ${ }^{102}$.

Ces sagesses se rejoignent, car l'image qui vient se trouve à la fois dans l'épicurisme et dans le stoïcisme. Mais Plotin se pose la question: «Il l'a plus encore que ceux qui disent: je suis heureux, même dans le taureau de Phalaris» ${ }^{103}$, objectant: «le dirait-il mille fois qu'il ne l'est pas». C'est là tout le sens de son propos: il y a des conditions du bonheur qui sont essentielles, et à côté de cela le vrai Bien, qu'il faut atteindre.

\section{La matière}

Derrière les apparences, se présente la vraie réalité, la structure intime des choses. Il y a deux choses, essentiellement, la matière et le vide, ou à côté d'un élément compact, les particules de matière, existe une autre réalité, le vide. Ces

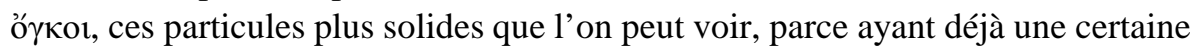
dimension, sont composées de réalités invisibles, que l'on ne peut plus diviser: les atomes ou particules microscopiques, que l'esprit peut concevoir, mais pas la vue. La structure de la matière est ainsi granulaire.

Dans cet enchevêtrement qu'est la matière, les atomes ont forme, grandeur et

\footnotetext{
98 Ennéades I, 4 (46), 2, 23

99 Ennéades I, 4 (46), 6, 4.

100 Ennéades I, 5 (36), 8, 5-6, cf. Lettre à Ménécée, 122.

101 Ennéades I, 4 (46), 4, 34-35;

102 Epicure, Maxime Capitale, $\mathrm{XL}$

103 Ennéades I, 4 (46), 15, 7-10, cf. Usener, Epicurea 60.
}

EMERITA (EM) LXXIV 2, julio-diciembre 2006 pp. 289-320 ISSN 0013-6662 
poids. Leur grandeur peut varier, mais contrairement à ce que dit Démocrite, pas à l'infini; leur forme est carrée, triangulaire, ronde ou oblongue. C'est leur poids qui les fait se mouvoir, puisqu'il y a entre eux, un vide qui rend possible se mouvement, lequel se fait en raison du poids, vers le bas. Mais, à la suite des chocs, il peut aller vers le haut, et dans tous les sens: aussi à un mouvement premier, s'ajoute ce deuxième consécutif au choc qui ralentit ou accélère leur vitesse, laquelle, malgré tout s'uniformise, dans l'ensemble. Cette réalité est difficile à concevoir, tout autant que ces infimes particules des atomes quasi indestructibles qui continuent à se mouvoir éternellement, dans le vide de l'espace. Les autres qualités, la couleur par exemple, ne sont pas aussi subjectives que l'avait dit Démocrite, mais si les corps n'ont d'autres propriétés que la forme, s'ils sont indéformables et impénétrables, ce qui est une des premières propriétés de la matière, il a celles-là, accidentelles, telles la couleur, la solidité, le toucher... qui ne viennent que de certains arrangements, et de certaines positions par rapport à la vue» ${ }^{104}$, certains étant plus compacts dans les couches profondes, d'autres moins, ce qui fait varier la dureté ou la couleur.

L'univers est infini; on sait la raison: «ce qui a limite a une extrémité, or cette dernière fait défaut, donc l'univers est infini» ${ }^{105}$. Et cela se tire de sa texture, c'est à dire de sa présence du vide infini, et des atomes en nombre, lui aussi infini ${ }^{106}$. L'espace lui-même est ainsi conçu, dans son infinité, dès lors que sa limitation marquerait l'arrêt des atomes qui le composent. Cette conception allait rompre avec tout un passé de tradition hellénique, et avec Parménide: la conception des atomistes engageait celle d'une pluralité des mondes. En effet, les mondes actuellement existant «sont le résultat, et le résidu d'une infinité d'ébauches d'univers», et «il y a séparé par des espaces vides une infinité d'autres mondes disséminés au hasard» ${ }^{107}$. Ainsi l'idée de Démocrite est-elle reprise dans la Lettre: «tous ces mondes et tous ces agrégats limités se différencient au sein des tourbillons grands et petits» ${ }^{108}$. Lucrèce en donne la raison: l'univers s'étend dans toutes les directions sans jamais rencontrer de limite, en sorte qu'à chaque fois que la matière est en quantité suffisante, on a la formation d'un monde, puisqu' aucune chose ne saurait être unique, ni la terre, ni le soleil, ni quoi que ce

104 Epicure, Lettre à Hérodote, 48, Plutarque, Contre Colotès, 1110c, =30 Usener, Epicurea.; Cf. Lucrèce, De la Nature, II, 757-809.

${ }_{105}$ Epicure, Lettre à Hérodote, 41.

106 Epicure, Lettre à Hérodote, 39.

107 Ch. Mugler, Devenir cyclique et pluralité des mondes, Paris, 1953, p. 156.

108 Epicure, Lettre à Hérodote, 73. 
soit ${ }^{109}$.

Comment passer à l'intérieur de chacun d'eux, de la matière qu'on ne voit pas, à la formation des corps qui eux sont visibles: «Parmi les corps, on doit distinguer les composés et ceux dont les composés sont faits: ces derniers corps sont insécables et immuables- il le faut pour que toutes choses ne se résolvent pas en non être, et pour qu'il y ait des réalités capables de subsister dans la dissolution des composés ${ }^{110}$. Ces corps sont pleins, mais cette dissolution - univers ou choses particulières - se fait toujours, et l'élément plein est fait de matière, c'est à dire d'atomes; la dissolution des univers est très lente, celle des corps plus rapide, en raison de leur constitution.

En fait un corps est un composé, ou agrégat; on en vient à la formation de ces composés comme résultat de collision: «la solidité qui leur appartient les fait rebondir après le choc ... ou leur enveloppement, par le composé, leur permet de reprendre leur position primitive» ${ }^{111}$. Lorsque les particules sont rares, on a les gaz, air, feu, denses, les solides. Lucrèce insiste sur la formation des liquides: l'huile ou le vin, et parle des germes pour les êtres vivants. La nature livre ses secrets: il semble qu'Epicure ait conçu un processus graduel, à partir de minimae partes, ou d' ö $\gamma$ кor; mais les corps composés, ainsi formés d'atomes réunis ${ }^{112}$, deviennent plus, des organismes, $\sigma u ́ \sigma \tau \eta \mu \alpha$. Lucrèce parlera, ainsi de la foudre comme mouvement en cohésion ${ }^{113}$. On a ainsi toutes les formes de textures, à partir de l'enchevêtrement ( $\pi \varepsilon \rho\left(\pi \lambda\right.$ okì), ou du contact et des vibrations ${ }^{114}$.

La matière de l'atome subsiste dans le temps. Le temps est cette réalité difficilement saisissable, dont l'essence en une représentation qui mesure le mouve-

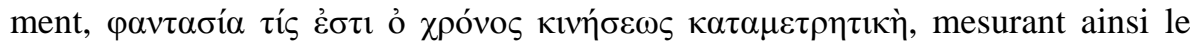
mouvement dans une conception proche d'Aristote, mais perçu à partir des jours et des nuits, il n'est pas seulement dépendant de ces mouvements célestes, mais concevable par la pensée ${ }^{115}$.

Dans ce monde des atomes et des corps, tous les mouvements semblaient s'inscrire dans un ordre naturel: nécessité implacable du devenir et du monde qui pour Démocrite est un principe de causalité, où «rien ne se produit fortuitement,

109 Lucrèce, De la Nature, II, 1048-49, cf. Ch. Mugler, op. cit., p. 180.

110 Epicure, Lettre à Hérodote, 41.

111 Epicure, Lettre à Hérodote, 44.

112 C.Bailey, The greek atomists and Epicurus, Oxford, 1928, p. 340.

113 Lucrèce, De la Nature, VI, 340-415.

114 Epicure, Lettre à Hérodote, 43.

115 Fragment 55 du Papyrus Herculanum 1413, cf. A.Barigazzi, «Il concetto del tempo nelle fisica atomistica», in Epicurea Bignone, Gênes, 1959, pp. 36-39.

EMERITA (EM) LXXIV 2, julio-diciembre $2006 \quad$ pp. 289-320 ISSN 0013-6662 
mais où tout procède de la raison et de la nécessité» ${ }^{116}$ Là où un «processus physique représentait le mécanisme des mouvements atomiques et comprenait ainsi, à la fois, la matière et le mouvement».

Ainsi arrive-t-on à cette curieuse doctrine du clinamen, ou déclinaison, ou encore déviation des atomes, qui concentre une partie des critiques, au point que certains émettent des doutes sur son existence ${ }^{117}$, dans la mesure où elle n'est pas présente dans la Lettre à Hérodote. En effet, si l'on dit: l'atome suit son mouvement, puis dévie un tout petit peu; tout est changé, à partir de là, il rencontre les autres, les chocs ont lieu, et la liberté est ainsi créée, on comprend le scepticisme, voire l'ironie de certains.

C'est pourquoi, il est nécessaire de présenter les choses autrement; car les détracteurs n'ont rien compris au matérialisme, lorsqu'ils affirment: «l'esprit ou l' âme conserve sa pleine indépendance» ${ }^{118}$; non pas. Dans une pensée idéaliste, la volonté peut être libre de dominer la matière, mais pour un matérialisme, rien n'est dans l'esprit ou dans l'âme qui ne soir auparavant dans la matière: aussi dirons-nous, Epicure se devait de créer le clinamen, ou à défaut, se confiait à un déterminisme intégral.

Il l'a fait, parce qu'il devait le faire: l'atome qui dévie est semblable à l'homme qui est fait de particules de matières; s'il dévie et s'il est libre, dans la matière, l'homme l'est. Sinon il reste inscrit dans ces lois physiques d'un déterminisme universel, le même qui détermine les trajectoires atomiques. La déviation de l'atome est une démonstration que la liberté est inscrite au cœur de l'être, elle prouve la facticité de la liberté.

On ne le connaît que de manière indirecte. Cicéron écrit: «Ainsi Epicure se rendait compte que si les atomes étaient entraînés vers le bas par leur propre poids, rien ne serait en notre pouvoir, puisque le mouvement serait déterminé et nécessaire; il trouve le moyen d'échapper à la nécessité...» ${ }^{119}$. Lucrèce l'explique, Philodème en parle, et encore Diogène d'Oenanda ${ }^{120}$.

116 Aetius, I, 25, 4 ( DK.68 B2), cité par P.-M. Morel, Atome et nécessité, Epicure, Lucrèce, Paris, 2000, p. 17.

117 Cf. M. Solovine, Epicure, Lettres et Maximes, Paris 1965, pp. 173-178.

118 M. Solovine, op. cit., p. 184.

119 Cicéron, La Nature des dieux, Belles Lettres, Paris, 2000, p. 31.

${ }^{120}$ Lucrèce, De la Nature, II 216-293, Philodème, De Signis, 54, Diogène d'Oenanda, Fgt 53, dans La philosophie épicurienne sur pierre, Fribourg, Paris, 1996, p. 51: «Ne sais tu pas donc qui que tu puisses être, qu'il y a une sorte de mouvement libre dans les atomes, que Démocrite n'a pas découvert, mais qu'Epicure fit connaître, étant une déclinaison, comme il le montre à partir des phénomènes» 
Epicure a pu la développer dans un autre texte plus tardif, puisque la déclinaison apparaît ainsi comme le produit d'une réflexion philosophique élaborée. On le voit dans les Fragments reconstitués du Papyrus Herculanensis 697, 1056 et 1191, du traité De la Nature d'Epicure qui était un traité d'école, où on a pu déchiffrer les phrases suivantes: «contre les arguments de cette sorte appelés nécessité», «à s'attribuer lui-même l'action et à maintenir la nécessité, il ne serait pas cohérent...» «il faut avoir affaire aux causes et ne pas tenir la nécessité pour responsable de tout» ${ }^{121}$. On comprend ainsi qu'Epicure devait trouver une solution au déterminisme universel, et qu'il inventa cette théorie de la déclinaison de l'atome, comme cette trace de la liberté humaine.

L'opposition de Plotin à Epicure, à propos de la matière, parât être radicale, tant il est vrai qu'on ne saurait réconcilier une doctrine issue du matérialisme, avec une vision qui part des idées et des formes, ou encore plus de l'Un, et se présente comme un idéalisme affirmé, avec cette autre.

La doctrine plotinienne de la matière ne saurait être envisagée qu'à partir de l'ensemble d'un monde divin, dont elle issue; encore faut-il, dans cet onto-théologie, et même cosmologie, voir ce qui prédomine. Certes la matière se présente comme l'élément dernier d'une descente, mais peut-on dire pour autant qu'elle a été créée? L'ambiguïté et l'entremêlement des positions est telle, qu'il est parfois difficile de se faire une idée; peut-être certaines ne sont-elles pas assez justifiées ${ }^{122}$, mais prétendre que la matière est quelque chose de créé, consisterait à dire que Plotin rejoint tout simplement les positions chrétiennes, ou gnostiques, à une époque où un siècle et demi plus tard, au moment du platonisme alexandrin, de Hiéroclès, ce sera le critère de séparation du platonisme et du christianisme ${ }^{123}$. Dans cette perspective créationniste, il reste fidèle, pour l'essentiel au Timée de Platon ${ }^{124}$. Ainsi la matière est-elle éternelle ${ }^{125}$ «est-elle toujours identique à ellemême, à ce qu'elle était au début», ainsi que le note $\mathrm{H}$. Benz ${ }^{126}$, et possède de la

121 Cf. D. Sedley, «Epicurus' refutations of determinism», in Syzêtêsis, Studi sull'epicureismo greco e romano, Mélanges M. Gigante, 1983, pp. 21-23.

${ }_{122}$ Cf. D. O'Brien, Plotinus, On the origin of matter, an exercise of the interpretation of the Enneads, Leiden, 1991.

${ }^{123}$ Cf. N. Aujoulat, Le néoplatonisme alexandrin, Hiéroclès d'Alexandrie, 1986, ou en I. Hadot, Le problème du néoplatonisme alexandrin, 1978, cf. notre «Ammonius et Plotin», Revue philosophique de Louvain, 102, p. 87.

${ }^{124}$ Cf. H.R. Schwyzer, «Zu Plotinus Deutung der sogennannten platonische Materie», Mélanges De Strycker, 1973, pp. 266-280.

125 Ennéades III, 6, (26), 10, 12; 11, 24; 13,29.

126 H. Benz, 'Materie' und Wahrnehmung in der Philosophie Plotins, Würzburg, 1990,

EMERITA (EM) LXXIV 2, julio-diciembre 2006 pp. 289-320 ISSN 0013-6662 
sorte cette identité à soi-même qui en fait quelque chose de pérenne.

Vient en effet, cette curieuse doctrine du traité I, 8, De l'origine des maux, que la matière c'est le mal. D'autant plus étonnante, qu'elle est le résultat de la procession de l'âme, telle qu'elle avait été décrite dans le traité IV, 8, De la descente de l'âme: «elle doit avancer toujours, jusqu'à ce que tous ses effets parviennent dans les limites du possible, au dernier des êtres, en raison de l'immensité de cette puissance qui s'étend à tous les êtres... si la nature de la matière est éternelle, il est impossible, puisqu'elle existe, qu'elle n'ait pas sa part du principe qui fournit le bien à chaque être, .... et si la production est une suite nécessaire, elle ne doit cependant pas être séparée de ce principe» ${ }^{127}$.

La procession n'a pas pour fin de créer le mal, et pourtant, il se produit: «Qu'est-ce que l'âme mauvaise... elle admet le mal, en allant au-delà ou en deça de la mesure; de l'intempérance, de la lâcheté, et tous les autres vices de l'âme» ${ }^{128}$. Et un peu plus loin: «Donc le premier mal, c'est la démesure; le deuxième mal c'est d'acquérir cette démesure, par ressemblance ou par participation, et de le prendre comme attribut. Car encore, le premier mal, c'est l'obscurité. Donc l'agresseur qui est le vice est la démesure de l'âme» ${ }^{129}$.

L'âme dans la procession a-t-elle fait preuve de faiblesse, en produisant la matière, et par là a-t-elle commis la faute: «... il reste qu'elle soit dans l'âme déchue qui n'est ni pure ni purifiée; et la faiblesse est pour cette âme, non pas la suppression de quelque chose qu'elle possède, mais l'addition d'un élément étranger» ${ }^{130}$. Mais on ne saurait confondre l'effet avec la cause: «c'est la matière qui est, pour l'âme, cause de faiblesse et de vice; c'est elle d'abord qui est mauvaise et qui est le premier mal» ${ }^{131}$.

Plotin, dans le traité, Contre les Gnostiques, envisage deux hypothèses: l'une, l'âme s'incline pour illuminer la matière qui existait déjà, et l'autre, c'est l'âme qui en s'inclinant produit la matière, et on en conclut: «si Plotin impose aux gnostiques une génération de la matière, c'est qu'il partage cette opinion ${ }^{132}$. Non pas. Il expose tout simplement différentes hypothèses, connaît les différentes théories du platonisme et du christianisme, dans le sens que nous venons

pp. 92-93.

127 Ennéades, IV, 8 (6), 6, 13-22.

128 Ennéades, I, 8 (51), 4, 5-10.

129 Ennéades, I, 8 (51), 8, 37-41.

130 Ennéades I, 8 (51), 14, 20-24.

131 Ennéades I, 8 (51), 14, 50-51.

132 Ennéades II, 9 (33), 12, 39-40, 41, D. O’Brien, Théodicée plotinienne, théodicée gnostique, Brill, Leiden, 1993, pp. 20-21.

EMERITA (EM) LXXIV 2, julio-diciembre 2006 pp. 289-320 ISSN 0013-6662 
d'indiquer, et les met à plat: «s'ils disent que l'âme l'a produite en s'inclinant, elle n'avait donc pas de raison de s'incliner, et la cause de l'inclinaison est alors non pas l'obscurité, mais la nature même de l'âme» ${ }^{133}$. Et lorsqu'il parle, luimême de production, il ne faut pas entendre celle-ci comme génération de la matière, au moins avec tout ce qu'on peut entendre dans le sens normal: «La procession du sensible, à partir de l'intelligible, note, J. Trouillard, se présente sous un aspect original, elle est très différente de celle du voũ s à partir de l'Un; elle est immanente aux âmes, car il n'y a pas production d'essences nouvelles, mais déroulement et désintégration de celle de l'esprit» ${ }^{134}$.

D'ailleurs II, 9 revient à un platonisme plus classique:«demander pourquoi le monde a été fait, c'est demander, pourquoi il y a une âme, et pourquoi le démiurge produit» ${ }^{135}$. Les vraies raisons du mal sont en la matière et non pas en l'âme. C'est ce note encore le traité I, 8: «Si elle est dans une chose, ou bien cette chose était déjà sans mesure, et puisqu'elle était déjà sans mesure, elle n'en a pas besoin ... Il faut donc qu'il y ait un illimité en soi» ${ }^{136}$. Et un peu après: «Le premier mal, c'est la démesure, le deuxième de l'acquérir par ressemblance ..., le premier mal, c'est l'obscurité» ${ }^{137}$.

En fait, elle est mauvaise parce qu'elle est illimitée: c'est à dire que cela renvoie à structure ontologique, c'est à dire à une essence qui explique sa disponibilité ultérieure pour le Bien ou le Mal; il n'y a là rien d'extraordinaire à expliquer la position éthique à partir d'une structure d'être. Et la doctrine serait inspirée du pythagorisme: «Numénius de l'école de Pythagore... a donné à Dieu le nom de monade, à la matière celle de dyade: cette dyade, d'après lui indéterminée n'a pas de génération déterminée, mais est engendrée» ${ }^{138}$.

Une autre raison serait le retour à un platonisme plus strict, du Sophiste ${ }^{139}$, car

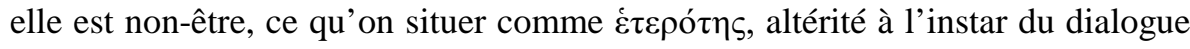
platonicien, et ainsi voir chacune des hypostases comme négation, et différence de l'autre; elle est autre que l'âme ${ }^{140}$. Mais, non-être relatif ou non-être absolu? En I, 8, Plotin déclare: «Il faut entendre ici non pas non être comme non-être ab-

133 Ennéades II, 9 (33), 12, 39-43.

134 J.Trouillard, La procession plotinienne, P.U.F, Paris, 1955, p. 12.

135 Ennéades, II, 9, 8, 1-2.

136 Ennéades I, 8 (51), 3, 27-30.

137 Ennéades I, 8 (51), 6, 37-40.

138 Numénius, Fragments, 1993, fgt 52, p. 95= Chalcidius, In Timaeum, C. 295.

139 Sophiste, 255 3-6.

140 Cf. K.H. Schlette, Das Eine und das Andere, Munich, 1966, pp. 131-133 «relationales mê on, relationales Negativität».

EMERITA (EM) LXXIV 2, julio-diciembre 2006 pp. 289-320 ISSN 0013-6662 
solu, mais seulement comme "cet autre que l'être", mais non pas comme sont non-être le mouvement et le repos qui se rapportent à l'être, mais plutôt comme une image de l'être ou comme quelque chose qui est encore plus non-être» ${ }^{141}$. Ce qui était la vision de VI, 9: «la nature de l'âme ne peut aller au non-être absolu; si elle descend vers le bas, elle va dans le mal, et ainsi dans le non-être, mais non pas dans le non-être absolu» ${ }^{142}$.

Mais d'autres textes rendront un autre son; ainsi en III, 6: «non-être, non pas au sens où l'on dit qu'ils ne sont pas dans l'être; c'est le vrai non-être» ${ }^{143}$. Et Plotin d'ajouter alors qu'elle est comme un reflet, un «fantôme», et qu'elle a toutes les apparences de l'illusion. Cela peut poser problème: ou la matière a une réalité ontologique, très faible, dérisoire, mais a quand même un être si infime soit-il, ou elle n'en a pas du tout probablement en raison du caractère illimité qui est le sien.

En sorte que la vision épicurienne ne trouve pas grâce aux yeux de Plotin, puisqu'elle en est à l'opposé, dans l'infiniment grand, même la déclinaison: «Il ne faut pas laisser place à de vaines déclinaisons, ni "à un mouvement subi des corps qui a lieu sans que rien ne le précède", ni à une inclinaison inconstante de l'âme, sans que rien ne l'ai poussée à faire ce qu'elle ne faisait pas avant» ${ }^{144}$.

Celle-ci ne produit pas la moindre liberté: elle laisse l'homme livré à un monde de la matière, sans plus, sans lui permettre quoi que ce soit d'autre: «Les uns posent des principes corporels, comme des atomes, pour eux le rapport des choses, leurs états comme leur naissance sont l'effet du mouvement des atomes, de leurs chocs et de leurs entrelacements, comme sont l'effet, la constitution, et les passions des choses: nos tendances et nos dispositions dépendent aussi de l'action de ces principes. Il y a là une nécessité qui résulte des atomes et que l'on introduit dans les êtres» ${ }^{145}$. La nécessité n'a donc pour Plotin pas été résolue: elle résulte du fait même de la matière qui a introduit un déterminisme, qui fait de nous des choses, dans nos états et notre naissance, en sorte que nous ne sommes plus maîtres de nous-mêmes, comme le reproche qu'il adressait aux astrologues, de nous transformer en tas de cailloux ${ }^{146}$.

L'atomisme d'Epicure marque le règne de la matière devenue ainsi le seul

141 Ennéades I, 8 (51), 3-9.

142 Ennéades VI, 9 (9), 11, 35-38, cf. M.-I. Santa-Cruz de Prunhes, La Genèse du monde sensible dans les Ennéades de Plotin, Vrin, Paris, 1979, pp. 101-102.

143 Ennéades, III, 6 (26), 7, 10-13, cf. M.I .Santa-Cruz de Prunhes, op. cit., p. 103.

144 Ennéades, III, 1, (3) 1, 16-19 = 280 Usener, Epicurea.

145 Ennéades, III, 1 (3), 2, 10-14., cf. Lettre à Hérodote, 40-41.

146 Sur ce point, cf. P. Henry, «Le problème de la liberté chez Plotin», Revue néoscolatique de philosophie, 33, 1931, pp. 50-74, 100-215, l'expression est de P. Henry. 
principe; Plotin parle de «principes corporels», alors que, s'il y a deux principes, pour lui, la matière, la dyade indéfinie et l'Un, c'est bien sûr, ce dernier qui l'emporte. Sans doute, l'étendue contribue-t-elle à la formation des corps ${ }^{147}$, mais «la matière est indéterminée, instable, par elle-même, sans cesse en mouvement vers la forme, docile à toute influence, elle se multiplie en se transportant de tous côtés, par un devenir incessant» ${ }^{148}$. Elle est ainsi devenue imprévisible; aussi lui faut-il la forme. Est-elle un substrat? ${ }^{149}$

Aussi le système plotinien fonctionne-t-il en sens inverse: le principe, c'est l'Un, dator formarum, ou l'Intelligence, ou encore l'âme, sûrement pas la matière. Plotin, à ce niveau, ne croit même pas aux données scientifiques d'Epicure: «ainsi à propos du temps, dira-t-il: il y a ceux qui en font un intervalle du mouvement, d'autres sa mesure, d'autres un accompagnement» ${ }^{150}$ où l'on peut reconnaître la position épicurienne. Ou encore à la fin du traité $\mathrm{Du}$ Ciel, II, 1: «On ne peut se baser sur les corps d'ici, pour discuter sur ceux de là-bas, parce que ces corps n'ont pas le même type d'âme qui maintient leur cohésion» ${ }^{151}$, où l'on voit un démenti à la Lettre à Hérodote qui disait qu'en partant des choses de la terre on pouvait arriver à raisonner sur les phénomènes célestes ${ }^{152}$. Pour Plotin, ce n'est pas possible, «les mondes n'ont pas le même type d'âme», ce qui est l'essentiel ${ }^{153}$.

\section{La sensation}

On sait comment la Lettre à Ménécée parle de la mort «privation de sensation» ${ }^{154}$. Celle-ci n'est possible que parce que l'âme s'en est allée qui l'a suscité. C'est dire qu'elle a une nature mortelle, faite d'atomes, mais auxquels s'ajoute du vide, comme dans tout composé, ce qui le rend comme toute nature matérielle

147 Ennéades, II, 4 (12), 12, 1.

148 Ennéades II, 4 (12), 11, 38-41.

149 Ennéades II, 4 (12), 13, 3.

150 Ennéades II, 7 (45), 7, 25-26, cf. Usener, 294.

151 Ennéades, II, 1 (40), 8, 21. p. 162.

152 Epicure, Lettre à Hérodote, 80, 3-5, cf. R. Dufour, Traité du Ciel, Vrin, Paris, 2003,

153 Ennéades V, 9 (5), 1, 3-4 et 7-8: «les uns en restent là et pensent que les choses sensibles sont les premières et les dernières...ceux d'entre eux qui ont une lueur de raison pensent que c'est là la sagesse, ils sont comme ces lourds oiseaux qui tiennent beaucoup de la terre et que leur poids rend incapable de s'élever très haut»

154 Epicure, Lettre à Ménécée, 124.

EMERITA (EM) LXXIV 2, julio-diciembre $2006 \quad$ pp. 289-320 ISSN 0013-6662 
destructible: l'âme est un mélange ( $\kappa \tilde{\alpha} \mu \alpha)$ qui impliquerait une juxtaposition d'éléments conservant leur nature ${ }^{155}$.

Celui-ci est-il fait de particules subtiles et fines $(\sigma \tilde{\omega} \mu \alpha \lambda \varepsilon \pi \tau \mathrm{\nu} \mu \varepsilon \rho \dot{\varepsilon} \varsigma)^{156}$ en fixant la composition qui viendraient à échapper à la mort? Le fait qu'on voit au sein de ces fines particules, essentiellement, le souffle, le feu, et l'air ${ }^{157}$, ce qui fait trois éléments rassemblés, d'autres textes ajoutant un quatrième, sans nom ${ }^{158}$, donnant la psychicité ${ }^{159}$, qui serait à la base de la sensation, principe de vie essentiel.

Ainsi, la sensation est-elle le fait de l'âme, ou peut-on la définir comme la réunion intime «de la vision et de la pensée» ${ }^{160}$, les deux exemples privilégiés étant ceux de la vue et de l'ouïe, mais dans les deux cas «quelque chose émane des objets entre eux et nous». La perception finalement serait cette sorte d'appréhension ultime, comme dans les images des dieux, où «l'on aboutit à une impression mécanique par photosensibilisation de l'esprit» ${ }^{161}$, pour saisir les images qui émanent de la divinité ${ }^{162}$.

Dans le cas des dieux, on avait pu avoir l'impression de deux types d'images, celles émanant des dieux, se précipitant en un flux continu rejoignaient celles des astres, et arrivaient à nous avec ces fragments extérieurs ${ }^{163}$, donnant cette impression étrange. D'une manière générale ce sont les répliques qu'Epicure appelle des simulacres, $\varepsilon$ í $\delta \omega \lambda \alpha$, qui forment la sensation, se détachant des composés, et conservant des corps leur structure. Ce n'est pas tout à fait la même chose qu'avec les dieux, puisqu' ici, il s'agit de corps véritables, mais «il existe des répliques de même forme que les solides, qui, par leur finesse, s'éloignent des corps apparents» ${ }^{164}$, conservant la même position qu'ils avaient, au départ.

Ainsi se produisent, dans le vide, plus ou moins vite, en fonction de la résistance, à une très grande vitesse, ces images faites de corpuscules extrêmement subtils et fins, se propageant dans l'air ou dans le vide, jusqu'au moment où elles

155 G. B. Kernferd, «La doctrine de l'âme chez Epicure», in L'épicurisme antique, Cahiers philosophiques de Strasbourg, 15, 2003, p. 270.

156 Epicure, Lettre à Hérodote, 63.

157 Epicure, Lettre à Hérodote, ibid.

158 Aetius, IV, 3, $11=315$ Usener.

159 G. B. Kernferd, art.cit., p. 263.

160 Epicure, Lettre à Hérodote, 49, 3.

161 J.Kany-Turpin, «Les images divines, Cicéron lecteur d'Epicure»,Revue philosophique, 1986, p. 56.

162 Cicéron, La Nature des dieux I, 29.

163 Cf. notes 39 et 41.

164 Epicure, Lettre à Hérodote, 46. 
rencontrent un sujet qui les saisit, et l'on obtiendra la sensation ${ }^{165}$, ainsi qu'on peut s'en rendre compte encore avec l'exemple de l'éclair et du tonnerre, propagés pour la vue et l'ouie, à des vitesses différentes. Ce qu'il y d'extraordinaire, dans cette conception, c'est l'idée d' «un flot qui s'échappe d'une partie quelconque des corps sur la longue étendue» d'un tissu serré, et qui conservent la

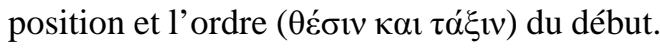

C'est probablement ce qu'il a voulu dire en exprimant l'opinion que «les sensations sont toutes vraies» ${ }^{166}$ qui là encore, étonna. Il semble que la difficulté de son interprétation ait arrêté Sextus Empiricus, au point qu'il ait conçu l'idée qu' «Epicure a voulu dire toutes les sensations existent (vँ $\pi \alpha \rho \chi 0 v) »{ }^{167}$, solution qui fut un temps celle des interprètes modernes ${ }^{168}$. Mais faut-il donner cette leçon affaiblie d'une phrase qui, comportait certainement une part de défi? Ou faut-il y voir une série de sophismes, tels une sensation ne peut pas en réfuter une semblable, une dissemblable une autre, ou la raison ne peut pas réfuter la sensation, même dans sa part d'irrationnel ${ }^{169}$. La leçon de ce dernier, serait ainsi: il faut être attentif aux sensations. Comme pédagogie, elle permet de préciser le sens de l'affirmation, dans une direction qui a ainsi échappé à la plupart des interprètes; toutes les sensations sont vraies signifierait: elles sont toutes porteuses d'une vérité dont il faut tenir compte.

Epicure serait ainsi tributaire d'un phénoménisme strict: la vérité de la sensation, c'est la vérité d'un phénomène physique, et la phrase était une invitation à reconnaître cette vérité du phénomène, en sorte qu'elle doit être respectée. Rien ne sert de la poser en terme moderne de connaissance d'un objet par un sujet. La sensation se déroule comme un film, avec l'envoi et la projection de ces petites particules appelées $\varepsilon^{\prime \prime} \delta \omega \lambda \alpha$ qui étaient elles même l'émanation de l'objet, dès lors qu'elles nous envoient un message qu'il faut reconnaître comme tel, comme la chaleur de la canicule, ou comme quand on dit: le ciel est bleu; rien ne servirait pour Epicure de corriger, il est noir; non, car des rayons lumineux le font apparaître bleu; c'est une vérité, comme déjà les simulacres ont pu un instant faire croire qu'il y avait des dieux de deux types; comme le bâton plongé dans l'eau

165 Epicure, Lettre à Hérodote, p..47.

166 Plutarque, Contre Colotès, 1109b.

167 Sextus Empiricus, M VII, 204, cf. G. Stricker, «Epicurus, On the truth of sense impressions», Archiv für Geschichte der Philosophie, 59, 1977, p. 126.

168 J.M.Rist, Epicurus, Cambridge, 1972, pp. 19-21.

169 Plutarque, Contre Colotès, 25. 1121c, Usener, 186, Cicéron, Des termes extrêmes des biens et des maux, I, 7, 22, pour le 2 è, Lettre à Hérodote, pour le 3 è, N.De Witt, «All sensations are true», TAPA, 74, 1943, p. 27.

EMERITA (EM) LXXIV 2, julio-diciembre 2006 pp. 289-320 ISSN 0013-6662 
paraît courbe: vérité à ce moment là. La vérité épicurienne est dans le temps, instantanée, et ponctuelle. On peut aussi dire qu'elle est dans l'espace, comme lorsqu'on énonce: la tour carrée est ronde, c'est une vérité à une certaine distance, non qu'il s'agisse d'une nouvelle vision à la Protagoras ${ }^{170}$, parce qu'elle n'est pas particulière, mais générale.

Les erreurs et les illusions viennent d'ailleurs: de l'interprétation fausse faite par le sujet, auxquelles il sera fait allusion dans la Lettre: «Le faux et l'erreur résident toujours, dans ce qui ajouté par l'opinion, qui doit être confirmé ou infirmé, mais qui ensuite ne l'est pas, en fonction d'un certain mouvement en nousmême dû à l'appréhension de l'image» ${ }^{171}$. Autrement dit, c'est une différence entre l'attente suscitée par la «prolepse», qui crée une opinion distincte de l'image. Lucrèce commenterad'abondance, au livre IV, les illusions des sens du navire qui nous porte, et semble en arrêt, des étoiles en mouvement, qui paraissent au repos, du soleil qui semble sortir de l'océan, sans compter les illusions des rêves: il ne faut pas imputer à la vue l'erreur de l'esprit ${ }^{172}$.

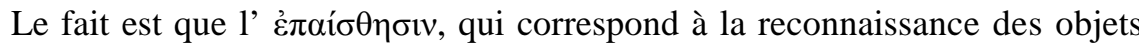
dans la Lettre ${ }^{173}$ ait une portée moindre que dans d'autres analyses de la perception, puisque la sensation s'est produite entre temps, avec toute sa force, venant s'agglutiner à l'esprit; rôle moins grand que dans le stoïcisme, ainsi qu'on le voit dans le traité de la sensation attribué à Philodème ${ }^{174}$ : dans ce texte transmis par le Papyrus Herculanum 19/698: «dans les différentes nuances, le discernement

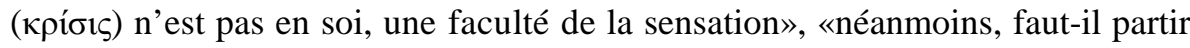
de la sensation, discernant par le toucher, ce qui est dur, mais se référant, pour le reste, au jugement: ainsi pour le chant» ${ }^{175}$. C'est à ce stade que peuvent venir les erreurs; mais ce n'est plus la sensation.

On s'attend à ce que Plotin s'attaque à la théorie matérialiste de la sensation, ainsi qu'il l'a fait à propos de la matière. Et, en effet, on voit apparaître plusieurs références au matérialisme stoïcien, dans les traités IV, 3-5, Des Difficultés relatives à l'âme. Ainsi, il se demande si l'âme serait capable d'imprimer en elle, les

170 Contrairement à N. De Witt, art. cit., p. 32.

171 Epicure, Lettre à Hérodote, 50, répété en 51-52.

172 Lucrèce, De la Nature, IV, 386, cf. 385-435.

173 Epicure, Lettre à Hérodote, 52.9.

174 A. Monet, «Philodème Sur la sensation», édition critique du papyrus Herculanum 19/698, Cronache Ercolanesi, 26, 1996, p. 27-126, et sur ce point, p. 65.

175 A. Monet, «Cratès, les normes et l'ouïe», dans Le jardin romain, épicurisme et poésie à Rome, Mélanges M.Bollack, P U.L. Lille, 2003, p 323.

EMERITA (EM) LXXIV 2, julio-diciembre 2006 pp. 289-320 ISSN 0013-6662 


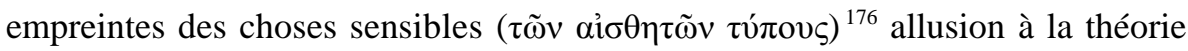
stoïcienne des empreintes reçues passivement, telle qu'elle pouvait se trouver en I, 3, «incapable de s'émouvoir de lui-même, mais disponible à toutes les impres-

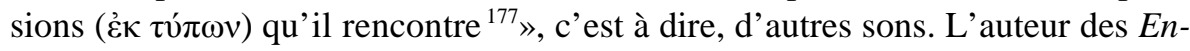
néades ne partage pas cette théorie de la sensation comme empreinte passive, et s'empresse de la désavouer.

Aussi ajoute-t-il juste après: «D'abord ces empreintes ne sont pas des grandeurs, il n'y a là «ni empreinte de sceaux, ni impression sur une matière résistante, ni modelage» parce qu'il n'y a pas davantage de choc, ni de surface enduite de cire» ${ }^{178}$. Il en est de même de la théorie stoïcienne selon laquelle l'âme se servirait de «la lumière comme d'un bâton pour atteindre l'objet visible, la sensation serait comme une action violente due à la résistance de l'objet et à la tension de la lumière ... De plus cet objet doit avoir été directement en contact avec nous» ${ }^{179}$.

Heureusement il y la théorie stoïcienne de la sympathie ${ }^{180}$, mais alors que dans cette théorie, la sympathie est une affection dépendant d'un $\pi v \varepsilon \tilde{\mu} \mu \alpha$, c'est à dire d'une combinaison matérielle élémentaire, il n'en parle plus que comme celle «qui existe entre les âmes sympathiques entre elles, parce qu'elles dérivent toutes d'une même âme, d'où vient aussi l'âme de l'univers» ${ }^{181}$; l'âme a remplacé le corps, et la sympathie est justifiée par la ressemblance entre deux êtres ${ }^{182}$, qui explique qu'ils ressentent en même temps la même chose ${ }^{183}$.

La sensation devient ainsi le fait de l'âme, et par là de l'intelligence: «la faculté de sentir et d'imaginer ont au-dessus d'elles la raison» ${ }^{184}$. Ainsi le rôle de l'âme est-il premier, et est-il pour la sensation celui d'un principe dirigeant, et unificateur, en raison de sa position: «elle est intermédiaire entre le sensible et l'intelligible, et dans cette situation elle peut se porter vers l'un comme vers l'autre» ${ }^{185}$. Et en raison de la nature humaine qui lui confie cette fonction et ce rôle: «La sensation est la perception des sensibles par l'âme et par l'animal,

176 Ennéades IV, 3, (27), 26, 28

177 Cf. notre Illusion de la dialectique et dialectique de l'Illusion, Paris, 2003, p. 425.

178 Ennéades IV, 3 (27), 26, 30-32, cf. SVF I, 484, II, 343; les chocs eux viseraient aussi Epicure.

179 Ennéades IV, 5, (29), 40-46.

180 Cf. A. Graeser, Plotinus and the stö̈cs, Leiden, 1972, pp. 68-72.

181 Ennéades IV, 3 (27), 1-3.

182 Ennéades IV, 5 (29), 1, 35-38.

183 Cf. E. K. Emillson, Plotinus, On sense perception, Cambridge, 1988, pp. 47-49.

184 Ennéades IV, 3 (27), 23, 21-23.

185 Ennéades IV, 4 (28), 3, 10-11.

EMERITA (EM) LXXIV 2, julio-diciembre 2006 pp. 289-320 ISSN 0013-6662 
lorsque l'âme saisit les qualités qui appartiennent à un corps, et imprime en elle la forme de ces corps» ${ }^{186}$. On insistera sur le fait que c'est alors l'âme qui réalise l'impression, en sorte qu'il y a connaissance par la sensation, «(lorsque) l'âme a donné au corps une trace d'elle-même» ${ }^{187}$. Le corps devenant ainsi son instrument: «Nous avons dit, note le traité De la vision, que celle-ci comme toute sensation s'opère par l'intermédiaire d'un corps...l'âme doit se mettre en contact avec les choses sensibles et employer des intermédiaires pour communiquer avec elles, par la connaissance et les affections» ${ }^{188}$.

(a) Ainsi la sensation devient-elle la perception des formes: «la différence des perception vient seulement de la différence des organes; toute perception est per-

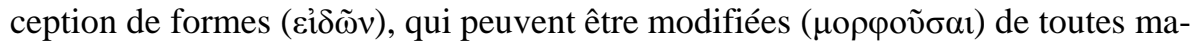
nières ${ }^{189}$. On note le curieux passage de la forme cĩioo $\varsigma$ à la forme $\mu$ ó $\varphi \eta$, la première, quand il s'agit de l'âme, la seconde à propos du sensible: «un objet visible vu de loin envoie une forme ( $\varepsilon^{i} \delta$ os): cette forme, à son début est bien indivisible; elle s'achève à son objet étendu, et l'âme voit la couleur et la forme telles qu'elles sont» ${ }^{190}$.

(b) Aussi la sensation est-elle comme dans le traité De l'Âme d'Aristote, une

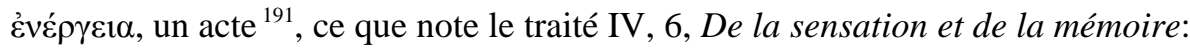
«la sensation juge d'objets qu'elle ne contient pas en elle; car il appartient à toute faculté de l'âme de ne pas subir des impressions, mais d'exercer sa puissance et son activité propre à propos des objets qui lui correspondent» ${ }^{192}$.

(c) Ainsi juge-t-elle et opère-t-elle un jugement: «chaque organe a ses impres-

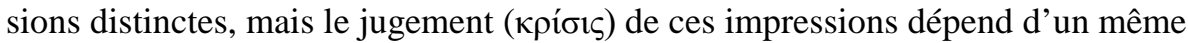
principe, qui, à la manière d'un juge, comprend les discours énoncés et les actes exécutés» ${ }^{193}$. Ainsi «la faculté de sentir est-elle une faculté de discernement» ${ }^{194}$. Rôle sans cesse affirmé: «c'est à l'âme avec sa faculté de juger qu'il appartient d'examiner le corps, et partant des affections du corps, d'énoncer ce jugement [qu'est la sensation]» ${ }^{195}$.

186 Ennéades IV, 4 (28), 23, 1-3.

187 Ennéades, IV, 4 (28), 20, 15-16.

188 Ennéades, IV, 5 (29), 2-4, et 5-10.

189 Ennéades, IV, 3 (27), 3, 17-19.

190 Ennéades, IV, 4 (28), 23, 15-18.

191 Aristote, De l'Âme, 426a, cf. E. K. Emilsson, op. cit. , pp. 126-133.

192 Ennéades IV, 6 (41), 2, 2-3.

193 Ennéades, IV, 3, (27), 3, 20-23.

194 Ennéades, IV, 3 (27), 23, 31-32.

195 Ennéades IV, 4 (28)22, 31-33, crochets d'E.Bréhier.

EMERITA (EM) LXXIV 2, julio-diciembre 2006 pp. 289-320 ISSN 0013-6662 
Dans le traité IV, 5, De la vision, que Plotin précise ses positions, après en avoir jeté les bases dans les deux précédents. Et l'on s'attend à y trouver une certaine opposition à Epicure, dès lors que la sensation procède non pas du corps, mais de l'âme se servant d'un corps, et qui fonctionne ainsi en sens pratiquement inverse de celui de l'épicurisme, par le privilège accordé à la préexistence d'une forme, et par le jugement limité chez celui-ci.

Or, lorsqu'il décrit le mécanisme de la vision, il s'attaque, à l'existence d'un milieu intermédiaire, des $\S 1$ à 3 , à partir de la question de savoir si celui-ci peut être considéré comme «un auxiliaire de la vision» ${ }^{196}$, après avoir situé celui-ci comme un corps dense. Or lorsque Plotin, en IV, 5, 1-3, essaie de mettre en cause que ce milieu soit l'air: «si la sensation est précédée d'une modification de l'air, en tournant nos regards vers lui, nous ne verrions pas l'objet, et nous sentirions seulement l'air situé auprès de l'œil» ${ }^{197}$, ce n'est probablement pas à Epicure et à Démocrite qu'il s'attaque, mais à une doctrine qui a été identifiée comme celle de Straton: «Straton dit que les couleurs sont transportées à partir des corps, en donnant la même couleur à un intermédiaire, l'air. Aristagoras que les formes font en impression sur l'air, à partir d'elles-mêmes» ${ }^{198}$ En sorte que s'attaquant à des doctrines intermédiaires, à la suite d'Alexandre d'Aphrodise, qui est en toile de fond de tout le commentaire, il ne s'adresserait qu'à des doctrines mineures, et non à Démocrite.

Certes, il fait une légère allusion à celui-ci en IV, 4: «à moins que l'on ne dise [avec Démocrite] que la couleur que l'on voit existe par convention et qu'il n'existe dans les objets, rien de pareil aux qualités» ${ }^{199}$. Pourquoi cela? Probablement parce qu'il reconnaît à la doctrine des atomes et du vide, et à la description épicurienne de la sensation, qu'il sait faite sur des bases démocritéennes - on a vu qu'il ne sépare pas les deux - une valeur scientifique. L'ensemble des trois traités est parti sur des bases scientifiques, à partir d'une allusion à Galien: «Et comme les nerfs ont leur point de départ dans le cerveau, on a placé dans le cerveau, le principe de la sensation et de l'appétition» ${ }^{200}$, et l'on sait que ses traités avaient eu un grand retentissement.

De même, sur le plan physique, de la matière, Plotin ne désavoue pas les don-

196 Ennéades IV, 5 (29), 1, 21.

197 Ennéades IV, 5, (29), 2, 50-52.

198 Aetius, IV, 13, 7-8, DG 403-404, cf. E. K. Emilsson, op. cit., p. 39, qui renvoie aussi à W.Theiler, Ennéades.

199 Ennéades, IV, 4 (28), 29, 32-33. [ ] de E.Bréhier.

200 Ennéades IV, 3 (27), 23, 9-12, cf. la note d'A.H.Armstrong, éd. Loeb, et de E.Bréhier, aux Ennéades.

EMERITA (EM) LXXIV 2, julio-diciembre 2006 pp. 289-320 ISSN 0013-6662 
nées scientifiques. C'est pourquoi, il respecte l'essentiel des positions démocritéennes et épicuriennes sur ce plan, et veut se mettre au diapason de celles-ci. Ainsi lorsqu'il dit: «les partisans des images [allusion à Epicure et Démocrite] disent que les images traversent le vide; ils exigent donc un espace libre, pour qu'elles ne soient pas arrêtées; et puisque l'obstacle est à son minimum, quand il n'y a pas de milieu, cette hypothèse ne conteste pas notre opinion» ${ }^{201}$. Plotin ne fonctionne pas dans le même registre: il écrit des traités philosophiques, ce qui lui permet de redonner force à l'âme et au jugement, mais il sait que ses positions n'auraient aucune valeur si elles démentaient celles de la science physique: aussi est-ce une sorte d'hommage leur opinion qu'il rend en disant qu'elles ne sont pas en désaccord avec les siennes.

Pour le reste, il essaiera de rejoindre celles du Timée de Platon, à même, pense-t-il de lui donner une assise comparable, $45 \mathrm{c}$-e, là où il s'agissait de la sensation qui est transportée du corps jusqu'à l'âme, mais surtout de ce feu qui jaillit de l'intérieur des yeux et rencontre celui des objets extérieurs» ${ }^{202}$. Plotin se demande ce qu'est «le rapport de la lumière contiguë à l'œil, avec celle qui est en lui et s'étend jusqu'à l'objet sensible» ${ }^{203}$, la vision devenant ainsi la relation d'une lumière, celle de l'oeil à une lumière, celle de l'objet.

Le problème de savoir si la sensation est un instrument fiable de connaissance est différent. Aussi n' y aura-t-il aucune contradiction à le voir désavouer sur ce point l'épicurisme, qui fait de celle-ci un instrument parfait. Aussi, au début de V, 5 s'attaque-t-il, non pas seulement aux épicuriens ${ }^{204}$, mais à tous ceux qui réduisent les objets de sensation à des impressions passives: «Mais, en admettant que les choses que la sensation nous fait percevoir ( $\alpha v \tau i ́ \lambda \eta \psi v v)$ sont bien dans les objet réels, ce qui est connu par le sens n'est qu'une image» ${ }^{205}$. Or le mot

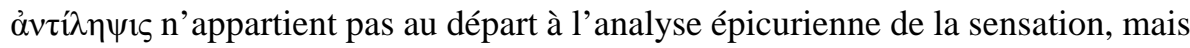
au stoïcisme: Philodème, dans le traité Sur la sensation, analysera précisément le terme d'appréhension, $\dot{\alpha} v \tau i ́ \lambda \eta \psi 1 \varsigma$, et de saisie: «là où il essaie de présenter un exposé de la théorie épicurienne, déterminé par la théorie stoïcienne de la sensation» ${ }^{206}$. Autrement dit ce mot était-il passé du stoïcisme à l'épicurisme plus

201 Ennéades IV, 5 (29), 2, 12-15. [ ] de nous.

202 Timée 45 c, cf. E. K. Emilsson, op. cit., p. 43, G. H. Clark, «Plotinus Theory of sensation», Philosophical Review, 51, 1952.

203 Ennéades, IV, 5 (29), 4, 1-2.

204 Contrairement à ce que dit E. Bréhier, Notice à V, 5, p. 83.

205 Ennéades V 5 (32), 1, 17-18.

206 A. Monet, op. cit, Cronache Ercolanesi, 26, 1996, p. 65 à propos de l'analyse de l'argument du traité de Philodème, col .IX-XVI du Papyrus Herculanum 19/698.

EMERITA (EM) LXXIV 2, julio-diciembre 2006 pp. 289-320 ISSN 0013-6662 
tardif. Aussi Plotin, en V, 5, s'attaque-t-il en premier lieu au stoïcisme, il est vrai qu'il poursuivra à l'encontre de l'épicurisme à propos du fait que la sensation puisse être conçue, dès qu'elle procède d'images, pour un critère fiable et permanent de la vérité, ce que concevait Epicure, et que Plotin réfute alors, non plus sur le plan d'une physique qui était celui d'Epicure, mais d'une théorie de la connaissance.

\section{Conclusion}

Aussi avons-nous voulu montrer dans cet article que la relation de Plotin à Epicure n'était pas seulement un relation doxographique, même si elle a pu être cela aussi ${ }^{207}$. Pour nous, même si Plotin a pu s'aider de temps à autre de manuels de citations, là n'est pas l'essentiel: la relation de Plotin à Epicure est une relation vécue et vivante d'une philosophie à une autre. Les travaux récents depuis H. Diels, mais surtout depuis un peu plus d'un quart de siècle ont beaucoup enrichi notre connaissance de Philodème, dans la Cronache Ercolanesi, mais aussi dans d'autres publications, et plus récemment ceux de Diogène d'Oeananda, en commentaire d'Epicure. Et si, a priori, rien ne peut faire envisager un rapport concret et vivant avec les épicuriens, on peut néanmoins dire que cette école existait toujours avec ses communautés essentiellement dans le sud de l'Italie, à l'époque de Plotin, où elles finiront après, par se dissoudre dans les communautés chrétiennes ${ }^{208}$. Si rien ne laisse, en effet préjuger d'un contact, peut-être parce qu'ils étaient les adversaires en raison de leur matérialisme essentiellement, on peut dire qu'ils étaient à l'horizon de la réflexion plotinienne. Car c'est comme cela qu'une pensée philosophique se dessine, dans ses contours essentiels, avec ses butoirs et ses engagements, l'ami et l'ennemi. Mais il arrive que l'adversaire fascine, et qu'on finit par partager un peu son point de vue. Est-ce le cas de ce traité 46, Sur le Bonheur, Plotin, vers la fin de sa vie a-t-il entendu quelque peu, une pensée qui en faisait le but de la vie, avec la plaisir? C'est pourquoi nous avons choisi de faire ce rapprochement et cette confrontation sur les quatre points qui nous paraissaient les plus essentiels: les dieux, la sagesse, la matière, la sensation.

Plotin ne partage pas cette théorie des dieux formés d'atomes et qui envoient des images, comme la Scolie de la Maxime les dépeint. Mais celle-ci ne paraît pas du tout la gêner, comme ne le gêne pas ces dieux qui ne négligent pas leur

\footnotetext{
207 Cf. l'article de J.-P. Dumont, «Plotin et la doxographie épicurienne», Les Cahiers de Fontenay, Néoplatonisme, Mélanges offerts à J. Trouillard, Mars, 1981, pp. 191-204.

208 Cf. G. Rodis-Lewis, op. cit., pp. 374-375.
} 
propre vie pour régler les affaires des hommes; seulement s'insurge-t-il avec véhémence contre l'abandon de la Providence.

Epicure avait été un libérateur sur le plan aussi de la sagesse, où on lui saura gré d'avoir proposé aux homme le choix d'une vie plus agréable. Est-ce ce qui suscite l'intérêt que nous avons de Plotin, pour la question du bonheur, et de savoir si le plaisir c'est le Bien; bien sûr il dira non, à la fin; celui-ci ne pouvant consister que dans le Bien.

Avec la matière l'opposition se fait plus se fait plus nette: l'univers matériel des atomes et du vide ne saurait lui convenir, peut-être parce qu'il avait une théorie de la matière, issue d'une procession, mais pour l'essentiel, dyade indéterminée s'opposant à l'Un, aux antipodes de celle-là, et surtout parce qu'il ne pouvait admettre que tout parte de la matière: il y avait le monde de l'esprit.

Avec la sensation, ses simulacres qui se dirigent vers l'œil, c'est encore autre chose, mais son opposition semble moins nette, au point qu'il affirme son accord à un moment avec les partisans de la théorie des images, ce qui est étonnant vu sa doctrine qui la fait partir de l'âme et privilégie le jugement, mais peut-être a-t-il vu dans ce côté scientifique de cette théorie de la sensation, qu'Epicure avait su par sa théorie des images saisir, en partie, une vérité du monde physique et des phénomènes.

Telle est la richesse de cette confrontation, ou par delà l'opposition massive décelée au stade de la matière du matérialisme et de l'idéalisme, certains rapprochements avaient pu se faire dans cette confrontation de deux horizons de pensée, pour lequel, on ne peut dire qu'Epicure n'ait en aucune façon compté dans la réflexion de Plotin. 